\title{
Long-term results of unilateral cleft lip repair with multiple infantile hemangiomas including one involving the cleft side of the upper lip
}

\author{
Dae Kyun Jeong ${ }^{1}$, Jae Woo Lee ${ }^{1}$, Soo Jong Choi ${ }^{1}$, Yong Chan Bae ${ }^{1,2}$ \\ ${ }^{1}$ Department of Plastic and Reconstructive Surgery, Pusan National University School of Medicine, Busan; ${ }^{2}$ Biomedical Research Institute, \\ Pusan National University Hospital, Busan, Korea
}

Infantile hemangiomas have a growth phase and an involution phase. For this reason, serial observation has generally been recommended as the treatment for uncomplicated infantile hemangiomas. Recently, however, individualized approaches have been emphasized. Although cleft lip and infantile hemangioma are common congenital diseases, infantile hemangiomas on the cleft side (i.e., in the operative field of the cleft lip) are extremely rare, and no clear guidelines have been established for their treatment. We experienced a case in which a patient with a cleft lip had an infantile hemangioma on the cleft side. In accordance with general treatment guidelines, cleft repair was performed 3 months after birth. The Millard rotation-advancement technique, which involves the use of a lower small triangular flap, was used for the repair. No intraoperative complications, such as massive bleeding, or postoperative complications were noted. The patient has received regular follow-up for the past 18 years, and other than a reddish scar on the lower lip, he currently has no related issues. Therefore, this case demonstrates that cleft lip repair performed according to cleft lip treatment guidelines produces good outcomes, even in cases involving a hemangioma on the cleft side.

Keywords Hemangioma / Cleft lip / Lip
Correspondence: Yong Chan Bae Department of Plastic and Reconstructive Surgery, Pusan National University School of Medicine, 179 Gudeok-ro, Seo-gu, Busan 49241, Korea

Tel: $+82-51-240-7273$

Fax: +82-51-243-9405

E-mail: baeyc2@hanmail.net

This article was presented at the Plastic Surgery The Meeting 2019 on September 20-23, 2019, in San Diego, CA, USA.

Received: March 30, 2019 • Revised: February 26, 2020 • Accepted: March 2, 2020

pISSN: 2234-6163・ elSSN: 2234-6171・ https://doi.org/10.5999/aps.2019.00395・Arch Plast Surg 2020;47:263-266

\section{INTRODUCTION}

The rate of cleft lip and cleft palate occurrence is roughly 1 in $700[1]$, and the global prevalence of infantile hemangioma is approximately $4 \%-5 \%[2]$. While these are relatively common deformities, infantile hemangioma on the cleft side of the cleft lip is extremely rare worldwide [3-5].

In accordance with the general cleft lip treatment guidelines, we performed cleft lip repair in a patient with infantile hemangioma on the cleft side of the cleft lip at 3 months after birth. The patient has received regular follow-up for the past 18 years.

\section{CASE}

This study was approved by the Ethics Committee of Pusan National University Hospital (IRB No. 2002-016-088). Informed consent was obtained for the use of patient data. The male patient was born at 39 gestational weeks with a birth weight of 2,500 g. Based on a physical examination, he was diagnosed with a left unilateral complete cleft lip with an alveolar cleft and 


\section{Fig. 1. Pediatric patient aged 3 months}

Infantile hemangiomas in the (A) lateral segment of the cleft side of the upper lip and lower lip, (B) helix of the right ear, and (C) left shoulder.
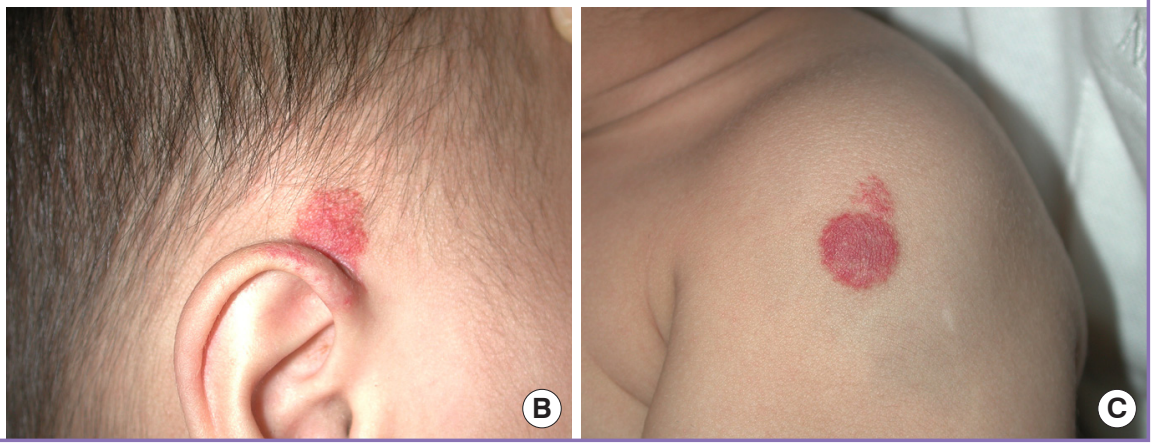

\section{Fig. 2. Six months after lip repair}

Residual infantile hemangioma was present in the repair site of the upper lip vermilion, and infantile hemangioma remained present at the lower lip.

a submucous cleft palate. Multiple infantile hemangiomas were found throughout his body, with no notable abnormalities observed in a chromosomal analysis.

Of particular note, the patient had infantile hemangiomas on the upper lip and lower lip, helix of the right ear, back, and left shoulder. The hemangioma on the upper lip was on the lateral segment of the cleft side (Fig. 1).

In accordance with the general guidelines for the treatment of cleft lip, cleft lip repair was performed at 3 months after birth. The Millard modified rotation-advancement technique, which involves the use of a lower small triangular flap, was chosen. The infantile hemangioma was ignored in the design, and unclear landmarks were determined. The operative field was infiltrated with 1:100,000 epinephrine-mixed lidocaine, and an incision was made with a \#15 scalpel. Sharp-tipped tissue scissors were used to separate the orbicularis oculi muscle from the skin and mucosa. The excised hemangioma was sent for a permanent biopsy, and the orbicularis oculi muscle, skin, and mucosa were
Fig. 3. Seven years after lip repair

Image taken immediately after correction of a cleft lip nose deformity. The infantile hemangioma at the repair site of the upper lip vermilion and lower lip was still not involuted.

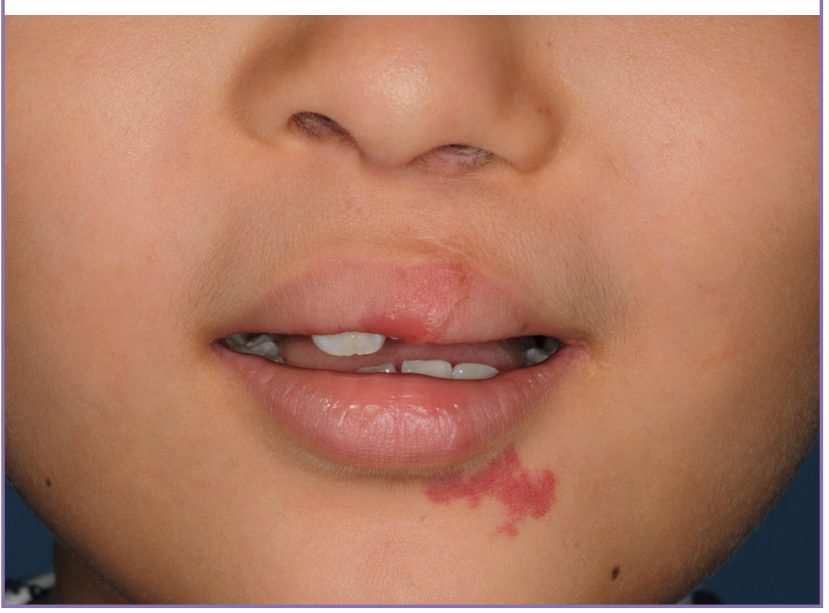

repaired. As the infantile hemangioma was ignored in the design, it remained present at the repair site.

There was no excessive intraoperative bleeding, and blood transfusion was not needed. The excised tissue was confirmed to be an infantile hemangioma upon histopathologic examination. The patient showed no abnormalities during recovery, and some residue of the infantile hemangioma remained at the repair site in the upper lip vermilion (Fig. 2).

Intravelar veloplasty was performed for the submucous cleft palate when the patient was 22 months old, and the patient was diagnosed with strabismus at the age of 3 years. At 5 years, the patient was diagnosed with mental retardation, right sensorineural hearing loss, and total hearing loss on the right side. At 7 years, the patient underwent correction for a cleft lip nose deformity (Fig. 3). At 9 years, he was diagnosed with a language disorder due to cleft palate and hearing impairment. At 11 years, an iliac bone graft was performed for the alveolar cleft. The residual infantile hemangioma in the repair site of the upper lip 


\section{Fig. 4. Eleven years after lip repair}

The hemangioma had been lost in the repair site of the upper lip vermilion. However, the infantile hemangioma on the lower lip was still not involuted.

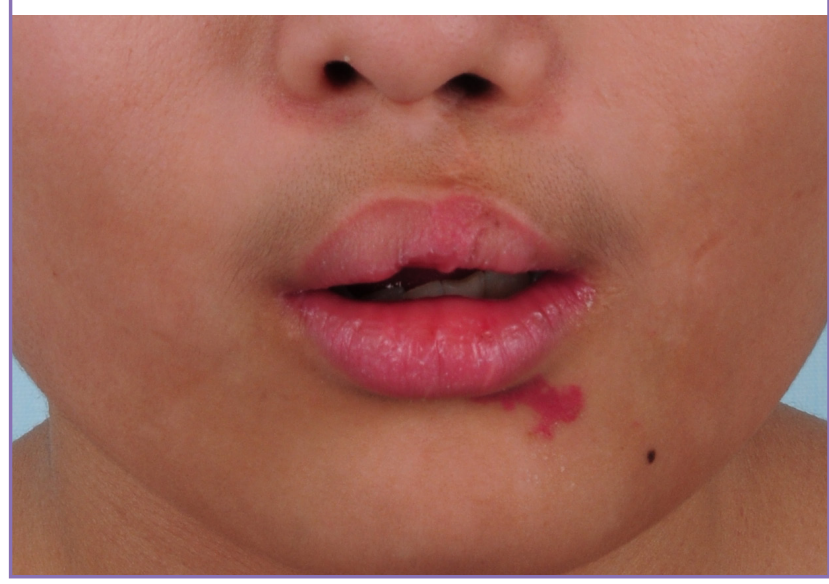

vermilion was involuted by the time the patient was 5 years old, and only a normal degree of scarring from the cleft lip repair was present. At the same time, the infantile hemangiomas in the helix of the right ear, back, and left shoulder were found to be completely involuted without any scarring (Fig. 4). The patient has received regular follow-up, and at 18 years old, he has no related problems other than a red scar on the lower lip (Fig. 5).

\section{DISCUSSION}

Infantile hemangioma is characterized by a growth phase and an involution phase, and serial observation is therefore recommended as the general treatment for uncomplicated infantile hemangiomas. However, individualized approaches may be utilized depending on the size, morphology, or location of the lesion, the presence or possibility of complications, the potential for scarring or disfigurement, the patient's age, and the rate of growth/involution $[6,7]$. Recent clinical guidelines published by the American Academy of Pediatrics reflect these trends and emphasize the importance of early intervention for infantile hemangioma [8].

An important determinant of the aesthetic results of cleft lip repair is the timing of the repair. Definitive cleft lip repair is often performed at 3-6 months of age in consideration of underlying medical issues, the need to minimize the risks of anesthesia, and the completion of pre-surgical orthopedic treatment [9]. Infantile hemangioma proceeds through a rapid growth phase for several months after birth, and slow growth may continue until 6-12 months after birth [10]. From then, the tumor shows improvement until 3-4 years of age and then ceases to improve [11]. Therefore, the opportunity for well-timed general

\section{Fig. 5. Eighteen years after lip repair}

Only a normal degree of cleft lip repair scarring could be observed in the upper lip repair site. On the lower lip, reddish pigmentation could still be observed.

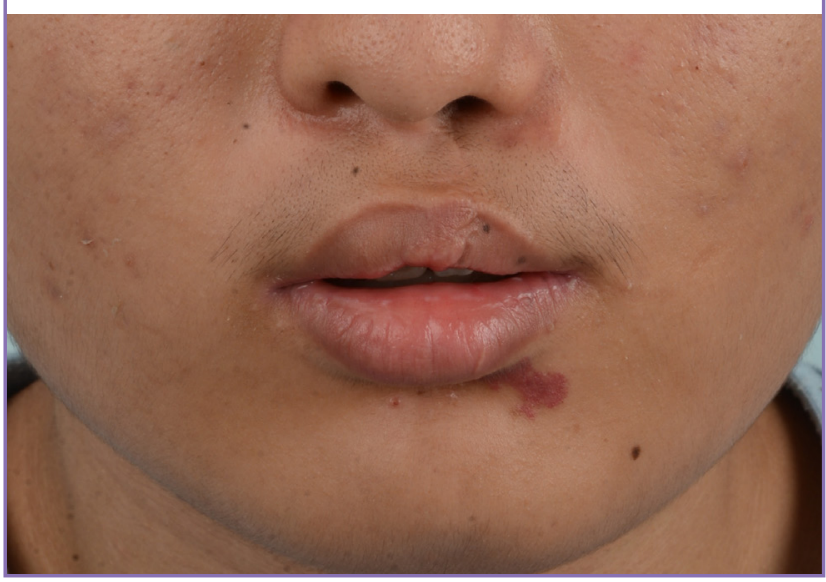

cleft lip repair may be missed if repair is delayed until the infantile hemangioma enters the involution phase.

Some factors that hinder early surgical intervention for infantile hemangiomas include anesthetic risks and the risk of bleeding due to the highly vascular nature of the tumor [12]. However, surgery may be a desirable option if early intervention makes later reconstruction easier [12]. Excising the hemangioma and repairing the cleft lip in early phases may also reduce cleft lip-related deformity. These recommendations are in line with recent trends in infantile hemangioma treatment. It is difficult to obtain optimal aesthetic results from cleft lip repair when it is delayed until after the infantile hemangioma is involuted. Further, potential psychosocial impairment among patients and caregivers resulting from delaying surgery until involution also cannot be ignored.

Yavuzer et al. [3] reported an early, single-stage cleft lip correction with partial hemangioma excision in a 3-month-old boy with a left unilateral complete cleft lip complicated by a hemangioma involving the lip, columella, and nasal tip. In typical cases of cleft lip, the philtral segment is shorter on the cleft side than on the non-cleft side, but in this case, due to tissue hypertrophy, the cleft-side philtrum was longer. Due to this finding, a back cut was not made on the columellar base. Instead, a 2-mm skin triangle was excised to equalize the non-cleft and non-hemangioma sides. In this procedure, the hemangioma in the repair site of the upper lip was totally excised. The recovery period was uneventful and without complications, and at 6-month followup, the lip showed satisfactory healing. In our case, the hemangioma showed less protrusion, and more of the anatomical landmarks of the cleft side of the upper lip were preserved. The infantile hemangioma was ignored in the design of the procedure, 
and unclear landmarks were determined. As a result, the hemangioma remained present at the repair site. No postoperative complications were observed at the repair site. Like the lesions at the other sites, the hemangioma at the repair site was involuted by the age of 5 years.

Bleeding risk is another factor that hinders surgical intervention for infantile hemangiomas. In our case, we performed cleft lip repair with adequate preparation for massive bleeding. However, no excessive bleeding occurred, and blood transfusion was not needed.

Infantile hemangiomas requiring lip repair for unilateral complete cleft lip are extremely rare, and no surgical guidelines have been established for this condition. In these cases, delaying lip repair until the infantile hemangioma is involuted may not lead to the best aesthetic outcome, and such a delay may also cause psychosocial impairment in patients and caregivers.

Therefore, we believe that general cleft lip repair produces good outcomes even in cases involving hemangiomas on the cleft side.

\section{NOTES}

\section{Conflict of interest}

No potential conflict of interest relevant to this article was reported.

\section{Ethical approval}

The study was approved by the Institutional Review Board of Pusan National University Hospital (IRB No. 2002-016-088) and performed in accordance with the principles of the Declaration of Helsinki. Written informed consent was obtained.

\section{Patient consent}

The patient provided written informed consent for the publication and the use of his images.

\section{Author contribution}

Conceptualization: YC Bae. Data curation: SJ Choi. Formal anlaysis: JW Lee. Methodology: DK Jeong. Projet administration: YC Bae. Vicualization: DK Jeong. Writing - original draft: DK Jeong. Writing - review \& edting: YC Bae. Approval of final manusccript: all authros.

\section{ORCID}

Dae Kyun Jeong https://orcid.org/0000-0002-6379-2629
Jae Woo Lee https://orcid.org/0000-0002-0945-6966 Soo Jong Choi https://orcid.org/0000-0002-8421-2945

Yong Chan Bae

\section{REFERENCES}

1. Mai CT, Cassell CH, Meyer RE, et al. Birth defects data from population-based birth defects surveillance programs in the United States, 2007 to 2011: highlighting orofacial clefts. Birth Defects Res A Clin Mol Teratol 2014;100:895904.

2. Kilcline C, Frieden IJ. Infantile hemangiomas: how common are they? A systematic review of the medical literature. Pediatr Dermatol 2008;25:168-73.

3. Yavuzer R, Latifoglu O, Ozmen S, et al. Unilateral cleft lip complicated by a hemangioma. Plast Reconstr Surg 2002; 110:1084-7.

4. Lo LJ, Noordhoff MS, Chen YR. Cleft lip and hemangioma: a patient with Wolf-Hirschhorn syndrome. Ann Plast Surg 1994;32:539-41.

5. Williams JK, Hitner JB, Wood RJ. Unilateral cleft lip repair in the presence of a vermilion hemangioma. Plast Reconstr Surg 1997;99:230-3.

6. Frieden IJ, Eichenfield LF, Esterly NB, et al. Guidelines of care for hemangiomas of infancy. American Academy of Dermatology Guidelines/Outcomes Committee. J Am Acad Dermatol 1997;37:631-7.

7. Frieden IJ. Which hemangiomas to treat--and how? Arch Dermatol 1997;133:1593-5.

8. Krowchuk DP, Frieden IJ, Mancini AJ, et al. Clinical practice guideline for the management of infantile hemangiomas. Pediatrics 2019;143:e20183475.

9. Parameters for evaluation and treatment of patients with cleft lip/palate or other craniofacial anomalies. American Cleft Palate-Craniofacial Association. March, 1993. Cleft Palate Craniofac J 1993;30 Suppl:S1-16.

10. Chang LC, Haggstrom AN, Drolet BA, et al. Growth characteristics of infantile hemangiomas: implications for management. Pediatrics 2008;122:360-7.

11. Couto RA, Maclellan RA, Zurakowski D, et al. Infantile hemangioma: clinical assessment of the involuting phase and implications for management. Plast Reconstr Surg 2012;130: 619-24.

12. Greene AK. Management of hemangiomas and other vascular tumors. Clin Plast Surg 2011;38:45-63. 